\title{
Rainfall Prediction with Agricultural Soil Analysis
}

\author{
Nidhi Kamble ${ }^{[1]}$, Darshan Ganeshpure ${ }^{[2]}$, Aditya Katte ${ }^{[3]}$, Prof. Aruna Pavate ${ }^{[4]}$ \\ ${ }^{[1],[2],[3]}$ Student of Information Technology, Atharva College of Engineering, Mumbai University-India. \\ ${ }^{[4]}$ Faculty of Information Technology, Atharva College of Engineering, Mumbai University-India.
}

\begin{abstract}
In India, the last drawback faced by the farmers is that the selection of correct yield for farming. Several factors influence the yield of crops like rain, temperature, soil, etc. Crop prediction helps farmers in choosing the right crop for plantation to maximize their earning. Prediction of crops could even be accurately through with the help of data mining techniques and considering the environmental parameters. During this work, the classifiers used area unit support vector machine and processing. Prediction of the crop is completed by considering parameters just like the quantity of rain, least and most temperature, soil type, humidity, and soil, $\mathrm{pH}$, scale price. the knowledge is collected from the agricultural website of geographical area. the knowledge is split into 9 agricultural zones. The associate interface has been designed through that farmers will enter the specified information to predict the crop. Classification of soil is required so that farmers can recognize the sort of soil and will plow the crops counting on the sort of soil.
\end{abstract}

Keywords - Rainfall Prediction, Agricultural, Soil Analysis, Data mining.

\section{INTRODUCTION}

Agriculture is the backbone of the Indian Economy. In India, the bulk of the farmers don't seem to be obtaining the expected crop yield due to many reasons. The agricultural yield primarily depends on atmospheric conditions. Rain conditions additionally influence rice cultivation. During this context, the farmers essentially need a timely recommendation to predict the end of the day crop productivity associate within the nursing analysis is to be created to help the farmers to maximize the crop production in their crops. Yield prediction may be a crucial agricultural drawback. Each farmer is fascinated by knowing, what proportion yield he's regarding expect. within the past, crop prediction was done by seeing grower's preceding knowledge on a selected crop. The degree of data is gigantic in Indian agriculture. data processing is widely applied to agronomic problems. data processing can analyse versatile data there's no restriction on the sort of knowledge [1]

Processing is used to research giant information sets and establish helpful classifications and patters within the knowledge sets. the overall goal of the knowledge mining method is to extract the knowledge from an information set and rework it into a perceivable structure for any use. during this paper, the foremost aim is to form a user-friendly interface for farmers, which supplies the analysis of rice production supported obtainable information. Completely different processing techniques are familiar with predict the crop yield for increasing crop productivity. data processing used for forecasting future trends of agricultural processes [2].

\section{LITERATURE REVIEW}

Farming is the most important part of the Indian (process of people making, selling, and buying things), still, it suffers from a heaping range of disasters like a natural process, unpredictable bad rainstorm or lack of it, (time periods with not enough rain), floods, moving (from one place to another) of farmers towards the cities in search of higher opportunities, and more. Individuals are concerned within the agriculture square measure the last taken care of, even once they square measure the one United Nations agency feeds the complete country. With establishments failing to support agriculture concerning providing loans and farmer welfare schemes, the time has returned for technology to need over the modification. Now each day the researchers, data analysts, and scientists have more targeting how mining and machine learning techniques are wont to analyse various soil profiles to complement the sector of agriculture [3].

Dr . Bharat Mishra, et al., [4] observed the research studies on the appliance of knowledge mining techniques within the field of agriculture. this is often accompany building digital maps for soil sorts and properties. Some people within the agricultural trade manage many acres of land, it's nearly impossible to urge prompt updates and alerts about potential issues while not ease from technology. Farmers Edge, a Canadian company takes daily satellite pictures of farms and combines it with different (clearly connected or related) information. It includes information from quite 4000 interconnected weather stations! (more than two, but not a lot of) countries like Eyre also/and depend on satellite-based soil and crop (instance of watching, noticing, or making a statement) to see areas tons of (quickly) than very old (success plans/ways of reaching goals) permit. This 
helps when the choice had been made what crops ought big on a selected piece of land. It saves an excellent deal of time and energy and results in higher yield production. Weather plays a very vital role in agriculture production and influence on the expansion, development, and yield of crops. A. Kumar (2004) [5] explains the techniques for forecasting of crops.

Weather aberrations will cause physical harm to crops and erosion. the quality of crops from the world to the market depends on the weather. Inclement will adversely have an impact on the quality of crops throughout transportation or storage. Data science consultant shrewdness to use tools that figure the patterns, and relationships that will rather be hidden. That they had finally decide something that push farming-based science forward through the check clearly stated/particular factors leading to change in weather. The findings caused by separation through (computer files full of information) and studies to shut things like this in the farming-based process will cause (excellent/very unusual) changes. Elements of farmingbased weather forecasts are: Amount and type of coverage of sky by the clouds, Rainfall and snow, max, min, and temperature temperatures, Relative wetness, Wind speed and direction, Low-pressure areas, cyclones, dangerous spinning wind storms, and depressions, Events like fog, frost, hail, (huge rainstorms with lightning and thunder), and wind strong winds/yells

Knowing the exact (material that makes plants grow better) rate might be a science and wishes a radical analysis of many factors. Often, many energetic/changing limits have forced the thought of. Such limits/guidelines hug/support crop nutrient uptake rates, analysis information, soil chemical, physical, and (related to the body function of living things) properties, weather, water (combination of different substances, objects, people, etc.), land type, soil testing (success plans/ways of reaching goals), cropwatering/rinsing with water ways of doing things, (material that makes plants grow better) (features/ qualities/ traits), interactions between (material that makes plants grow better)s and many of due to the standard of finding the "best fertilization range," (wrong and bad use of) (material that makes plants grow better)s might be a world development. most farmers still believe trying things that sometimes did not work, idea, and guess. The result is, crops don't meet their yield potential, and increase environmental pollution. informatics professionals' square measure now ready to tell the farmers with the right amount of fertilizers. The discovered knowledge is visually presented to the user [6]

In agriculture advanced algorithms' area units wanted to make a decision the patterns and behaviour of nature that helps in prediction invade pests and thus, the unfold of microscopic diseases. Classify soils can be started from the point of view of soils as a matter and soil as a resource [7].
Farmers need to manage pests. Digital tools and knowledge analysis in agriculture square measure getting used to scientifically handle harmful insects. Agricultural pests will quickly withdraw a farmer's profits but misusing pesticides will have adverse effects on people, plants, and different living things. Luckily, some firms have recruited informatics professionals to develop user-facing platforms that analyse once to use pesticides, and therefore the way tons of to use. While some insects may improbably helpful to farmers and thus, the crops, others may deadly and unfold diseases.

Climate change might be a looming concern that has already affected the agriculture sector. However, informatics consultants operating arduous to figure out ways to catch abreast of the change. The country's prosperity is depending upon the soils of that country [8].

One project involves giving IoT sensors to Taiwanese farmers of rice production so that they're going to collect information that's necessary about their crops. It'll help farmers to improve (as much as possible) their production cycles, though (related to the Earth's weather) changes create it very hard. the usual farming calendar isn't any sparer due to the extreme climate changes. However, information analysis will (totally change and improve) the end of the day of farming. Data scientists \{are additionally also are analysing agriculture soil information to understand, however, the soil will address \{climate change global natural process temperature change $\}$ by cathartic greenhouse gases, and however, the soil will adapt to global climate change.

We can additionally use weather prediction within the machine-controlled irrigation system. However, exactly? Having a system that tells you the soil is dry however you don't need to compelled to irrigate as a result it's going to rain once some hours look to be an interesting use case. All the countries within the planet square measure presently in an exceeding scenario wherever they're needed to use water in an exceedingly very economical way. keep with the recent studies, water is popping into tons of and tons of in short provides worldwide and fierce of the world population would face total water shortage by the year 2025. In agriculture too, the foremost downside that farmers face is that water insufficiency, thus reinforcing the usage of water, one of the irrigation systems victimization drip irrigation that enforces as a machine-controlled irrigation system for small scale farms. Another irrigation system machinecontrolled irrigation system victimization weather prediction. The natural classification of the soils is proposed by the Unified Soil arrangement (USCS) [9].

To improve soil's quality, we must know the current $\mathrm{pH}$ levels of your soil. Fertility levels of the principal nutrients. Type and measure of lime your soil needs. Nutrients got to add to your soil as fertilizer. Amount of fertilizer your crop and soil needs. Soil analysis can allow you to know where your soil needs treatment or improvement. this technique can do things like tell you 
which of the crop to grow. Prevent poor crops caused by drought, disease, insects, an excessive amount of water, or other problems. Substitute for correct cultural practices.

\section{METHODOLOGY}

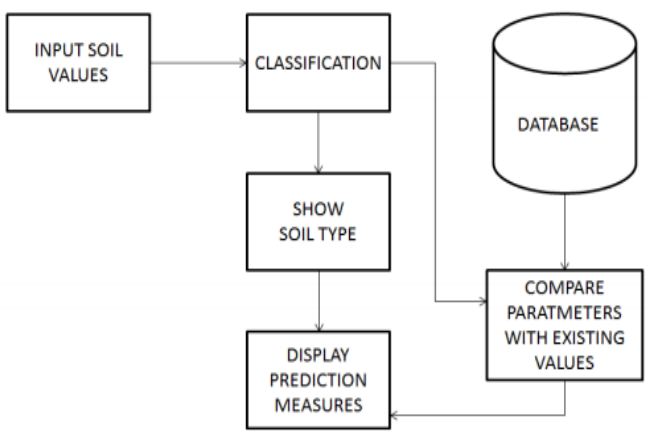

Figure 1: Block Diagram of Rainfall \& Soil Analysis System

The primary aim of this method is to classify the soil per the nutrients into it. For this, we have got taken datasets of soil samples. The soil goes to classified victimization call tree algorithm and type of soil getting to display. we tend to square measure getting to predict the crops proper for the particular sort of soil. Additionally, to the present, we do tend to square measure getting to improve the soil if the farmer desires to yield explicit crop within an equivalent soil by suggesting the requirements of the nutrients for the same soil. ID3 (Iterative Dichotomiser 3) employed to make call trees supported the categories (e.g. low, high, medium). This data is used by Naïve Bayes to teach the algorithm and apply it to a take a glance at file. Attributes square measure classified in ID3, into many categories like low, medium, or high. The nodes heavy categories' square measure called "decision nodes." the child nodes which will not any divided (leaf nodes), area unit, "classes" thereto the attributes belong, given condition. As an example, if $\mathrm{N}$-value (Nitrogen-value) may be a smaller amount than forty p.c it's classified as "low." Here, N-value is that the calling node and "low" is that the category thereto to the soil sample belongs. Entropy may do of however ordered file set is. Hence, the amount of knowledge needed for classification is directly proportional to an increase in Entropy. Entropy, need to thus be as low as potential. This is often (accomplished or gained with effort) by filtering the knowledge manually before providing it as a response to the set of computer instructions. Information is that the opposite of (the breakdown or decline of something into random disorder). Data gain (I. G) is that the price left when classifying the events. it's the excellence between (the breakdown or decline of something into random disorder) when the classification and before.

\section{Algorithm:}

Step 1: Calculate (the breakdown or decline of something into random disorder) of each attribute using the info set
Step 2: Split the set into subsets using the attribute that (the breakdown or decline of something into random disorder) may be a minimum

Step 3: Make a choice tree node containing that attribute Step 4: Recurs on subsets using remaining attributes ID3 algorithm imperatively classifies all the info.

\section{RESULT \& ANALYSIS}

The performance testing has been performed on testing datasets, and we merely used crop datasets to evaluate our system. Figures 8, 9, and 10 show the Weather information, Prediction of Soil Quality, and Prediction crop using rain\& fertility, respectively. Accuracy can be obtained by sum of True classified cases (TP) and True No classified cases (TN) divided by the total number of instances which is given in Eq.1

Accuracy $=(\mathrm{TP}+\mathrm{TN}) /$ Total number of Instances $(1)$

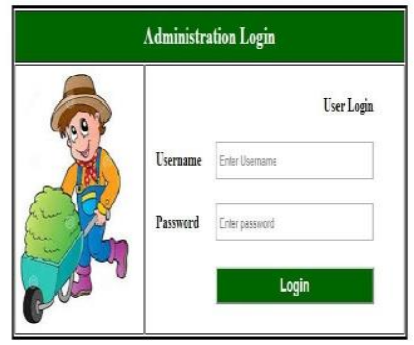

Figure 2: Administration Login

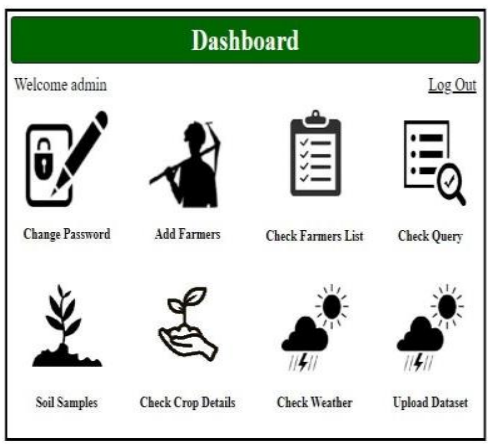

Figure 3: Dashboard 


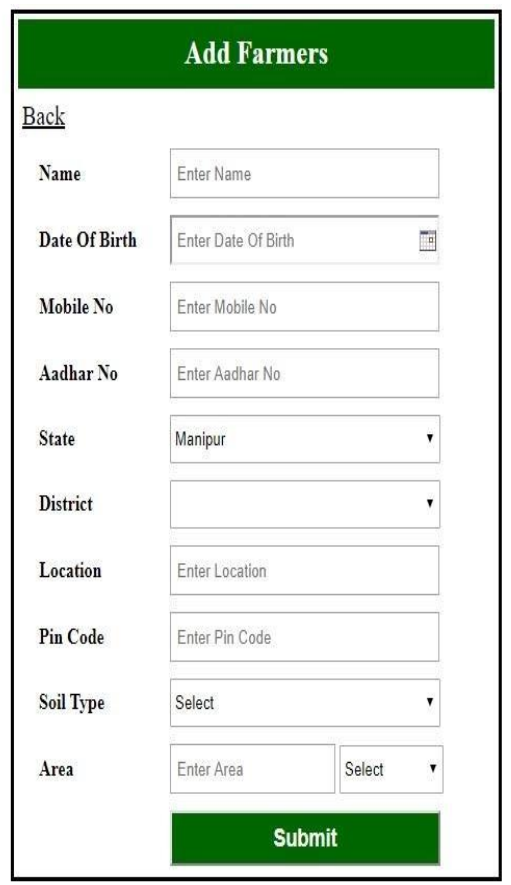

Figure 4: Add Farmers

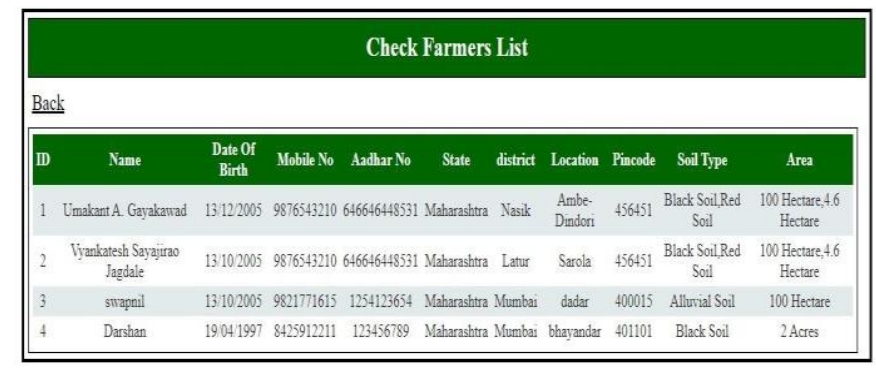

Figure 5: Check farmers List

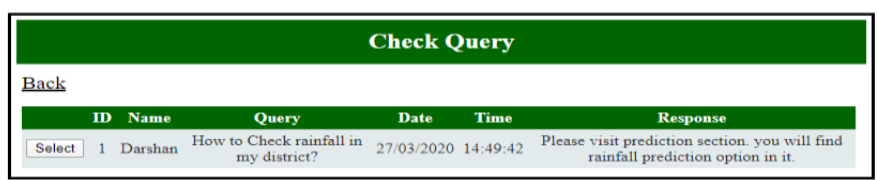

Figure 6: Check Query

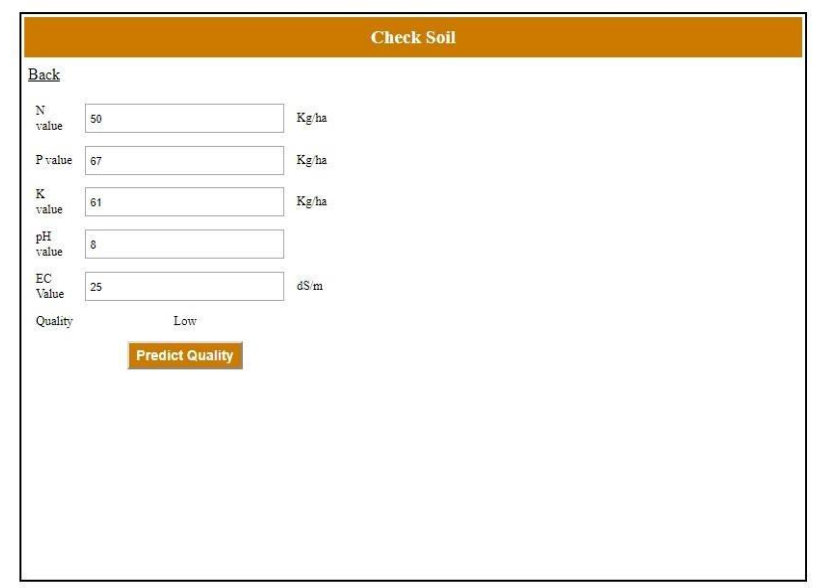

Figure 7: Check Soil

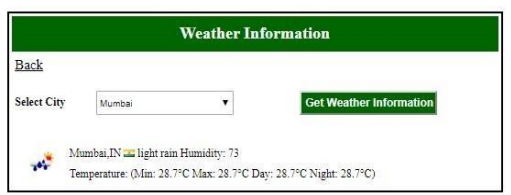

Figure 8: Weather Information

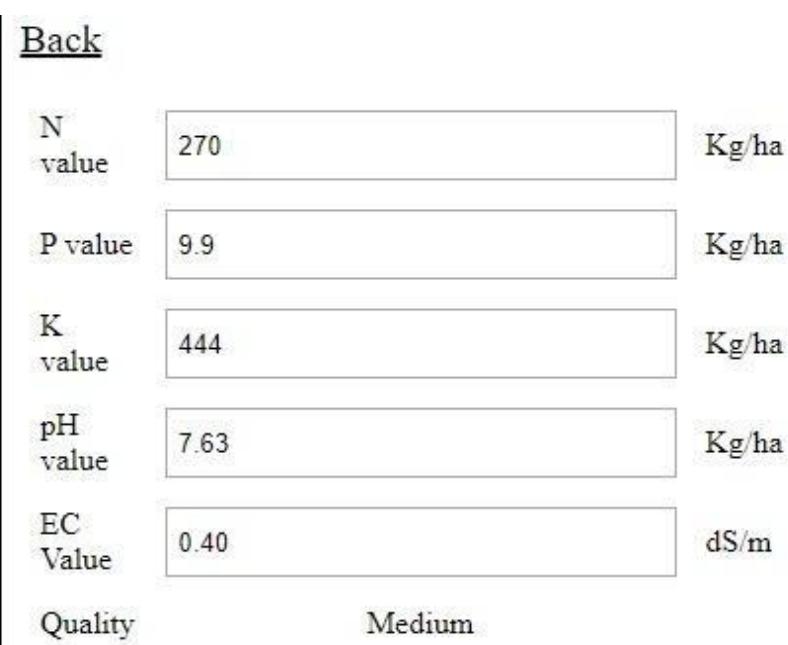

\section{Predict Quality}

Figure 9: Prediction of Soil Quality 


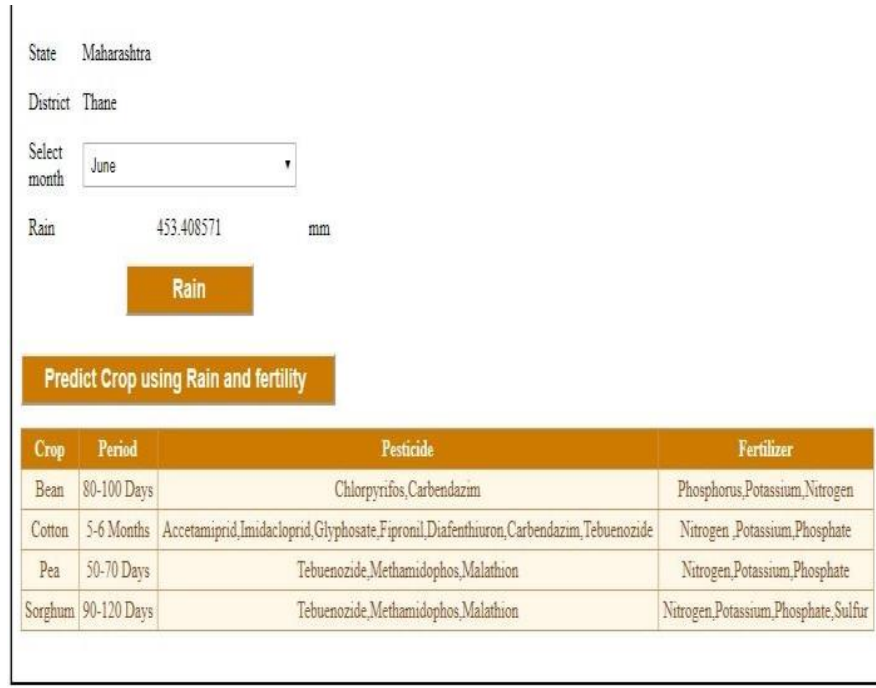

Figure 10: Prediction crop using rain \& fertility

\section{CONCLUSION}

This paper presents an analysis of the soil knowledge \& treatment of different sets of computer instructions and (statement about a possible future event) ways of doing things. This system can suggest related (material that makes plants grow better) for the given soil sample and cropping pattern. the best Indian areas' square measure chosen for the end of the day (rain, snow, etc.) analysis. Testing showed that the results of the proposed system are best. The longer term work mainly focuses on improving the farm-related area.

\section{REFERENCES}

[1]. M.C.S.Geetha, -A Survey on Data Mining Techniques in Agriculturell Vol. 3, Issue 2

[2]. Hetal Patel Research Scholar Charusat, Dharmendra Patel Assistant Professor Charusat, Changa in (2014) A Brief survey of Data Mining Techniques Applied to Agricultural Data.

[3]. V. Ramesh and K. Ramr Classification of agricultural land soils: A data mining approach International Journal on Computer Science and Engineering (IJCSE) ISSN : 09753397 Vol. 3 No. 1 Jan 2011379

[4]. S.Veenadhari, Dr. Bharat Misra, Dr. CD Singh, —Data mining Techniques for Predicting Crop Productivity - A review articlell, International Journal of Computer Science and technology, march 2011

[5]. Amrender Kumar, -WEATHER BASED CROP FORECASTING TECHNIQUES\|, Agricultural Statistics Research Institute
[6]. International Journal of Emerging Technology and Advanced Engineering Website: www.ijetae.com (ISSN 2250-2459, Volume 2, Issue 2, February 2012) 275 - Survey on Data Mining - VibhaMaduskar and Prof. yashovardhankelkar

[7]. Leisa J. Armstrong, Dean Diepeveen, Rowan Maddern - The application of data mining techniques to characterize agricultural soil profiles|

[8].[Online] Available from:

http://www.yourarticlelibrary.com/soil/soils-of-india-sixdifferent-types-of-soils-found-in-india/12779/

[9]. Isbell, R. F. (1996). The Australian Soil Classification. Australian soil and land survey handbook. (Vol. 4). Collingwood, Victoria, Australia: CSIRO Publishing. 2250-2459, Volume 2, Issue 2, February 2012) 275 - Survey on Data Mining - VibhaMaduskar and Prof. yashovardhankelkar

[7]. Leisa J. Armstrong, Dean Diepeveen, Rowan Maddern - The application of data mining techniques to characterize agricultural soil profiles|

[8].[Online] Available from:

http://www.yourarticlelibrary.com/soil/soils-of-india-sixdifferent-types-of-soils-found-in-india/12779/

[9]. Isbell, R. F. (1996). The Australian Soil Classification. Australian soil and land survey handbook. (Vol. 4). Collingwood, Victoria, Australia: CSIRO Publishing. 